Proceedings

Vol. , No. () 1-10

(C) World Scientific Publishing Company

\title{
THE NEW EARTH GRAVITY MODELS AND THE MEASUREMENT OF THE LENSE-THIRRING EFFECT
}

\author{
LORENZO IORIO \\ Dipartimento di Fisica dell'Università di Bari \\ Via Amendola 173, 70126, Bari, Italy
}

\begin{abstract}
We examine how the new forthcoming Earth gravity models from the CHAMP and, especially, GRACE missions could improve the measurement of the general relativistic Lense-Thirring effect according to the various kinds of observables which could be adopted. In a very preliminary way, we use the recently released EIGEN2 CHAMP-only and GRACE01S GRACE-only Earth gravity models in order to assess the impact of the mismodelling in the even zonal harmonic coefficients of geopotential which represents one of the major sources of systematic errors in this kind of measurement.
\end{abstract}

\section{Introduction}

The post-Newtonian gravitomagnetic Lense-Thirring effect ${ }^{1,2,3}$, in the slow motion and weak field approximation of the General Theory of Relativity, consists of secular precessions of the longitude of the ascending node $\Omega$ and the argument of pericentre ${ }^{a}$ $\omega$ of the orbit of a test particle freely falling in the gravitational field of a central spinning object of mass $M$ and proper angular momentum $J$

$$
\begin{aligned}
& \dot{\Omega}_{\mathrm{LT}}=\frac{2 G J}{c^{2} a^{3}\left(1-e^{2}\right)^{\frac{3}{2}}}, \\
& \dot{\omega}_{\mathrm{LT}}=-\frac{6 G J \cos i}{c^{2} a^{3}\left(1-e^{2}\right)^{\frac{3}{2}}},
\end{aligned}
$$

where $a, e$ and $i$ are the semimajor axis, the eccentricity and the inclination, respectively, of the orbit of the test particle and $G$ is the Newtonian gravitational constant; $c$ is the speed of light in vacuum.

\subsection{The LAGEOS-LAGEOS II Lense-Thirring experiment}

The first attempts to measure such effect in the gravitational field of Earth are due to Ciufolini and coworkers which analysed the data of the orbits of the existing LAGEOS and LAGEOS II geodetic satellites collected with the Satellite Laser

a In the original paper by Lense and Thirring the longitude of the pericentre $\varpi=\Omega+\omega$ is used instead of $\omega$. 
Ranging (SLR) technique ${ }^{4}$. The observable used is the following combination ${ }^{5}$ of the orbital residuals of the nodes of LAGEOS and LAGEOS II and the perigee of LAGEOS II

$$
\delta \dot{\Omega}^{\mathrm{LAGEOS}}+c_{1} \delta \dot{\Omega}^{\mathrm{LAGEOS} \mathrm{II}}+c_{2} \delta \dot{\omega}^{\mathrm{LAGEOS} \mathrm{II}} \sim 60.2 \mu_{\mathrm{LT}},
$$

with

$$
c_{1}=0.304, c_{1}=-0.350,
$$

The quantity $\mu_{\mathrm{LT}}$ is a solved-for least square parameter which is 0 in Newtonian

Table 1. Orbital parameters of LAGEOS, LAGEOS II, LARES, S1 and S2.

\begin{tabular}{lccccc}
\hline Orbital parameter & LAGEOS & LAGEOS II & LARES & S1 & S2 \\
\hline$a(\mathrm{~km})$ & 12270 & 12163 & 12270 & 12000 & 12000 \\
$e$ & 0.0045 & 0.014 & 0.04 & 0.05 & 0.05 \\
$i(\mathrm{deg})$ & 110 & 52.65 & 70 & 63.4 & 116.6 \\
\hline
\end{tabular}

mechanics and 1 in the General Theory of Relativity. The gravitomagnetic signature is a linear trend with a slope of 60.2 milliarcseconds per year $\left(\right.$ mas $\mathrm{yr}^{-1}$ in the following). The claimed total accuracy is of the order of $20 \%-30 \%$. In Table 1 we quote the orbital parameters of the existing or proposed LAGEOS-like satellites useful for general relativistic tests.

The main sources of systematic errors in this experiment are

- the unavoidable aliasing effect due to the mismodelling in the classical secular precessions induced on $\Omega$ and $\omega$ by the even $(\ell=2,4,6, \ldots)$ zonal $(m=0)$ coefficients $^{\mathrm{b}} J_{\ell}$ of the multipolar expansion ${ }^{\mathrm{c}}$ of the terrestrial gravitational field

- the non-gravitational perturbations affecting especially the perigee of LAGEOS II. Their impact on the proposed measurement is difficult to be reliably assessed

It turns out that the mismodelled classical precessions due to the first two even zonal harmonics of the geopotential $J_{2}$ and $J_{4}$ are the most insidious source of error in the measurement of the Lense-Thirring effect with the nodes of LAGEOS and LAGEOS II and the perigee of LAGEOS II only. It is so also because of their secular variations $\dot{J}_{2}, \dot{J}_{4}$, which can be accounted for by an effective $J_{2}$ coefficient $\dot{J}_{2}^{\text {eff }} \sim \dot{J}_{2}+0.371 \dot{J}_{4}+0.079 \dot{J}_{6}+0.006 \dot{J}_{8}-0.003 \dot{J}_{10} \ldots$, and of the fact that the LenseThirring effect is embedded in their recovered values released by any Earth gravity

\footnotetext{
${ }^{\mathrm{b}} J_{\ell} \equiv-C_{\ell 0}, \ell=2,4,6, \ldots$ The unnormalized Stokes coefficients $C_{\ell m}$ of degree $\ell$ and order $m$ can be obtained from the normalized Stokes coefficients $\bar{C}_{\ell m}$ according to $C_{\ell m}=N_{\ell m} \bar{C}_{\ell m}$ where $N_{\ell m}=\left[\frac{(2 \ell+1)\left(2-\delta_{0 m}\right)(\ell-m) !}{(\ell+m) !}\right]^{\frac{1}{2}}$

${ }^{\mathrm{c}}$ It accounts for the oblateness of Earth generated by its diurnal rotation.
} 
models since the gravitomagnetic acceleration is a priori included in the force models used in obtaining, among other things, the even zonal coefficients. The combination of eq. (3) is insensitive just to $J_{2}$ and $J_{4}$. According to the full covariance matrix of the EGM96 gravity model ${ }^{6}$, the error due to the remaining uncancelled even zonal harmonics amounts to almost ${ }^{7} 13 \%$. However, if the correlations among the even zonal harmonic coefficients are neglected and the variance matrix, in a root-sumsquare fashion, is used ${ }^{\mathrm{d}}$, the error due to the even zonal harmonics of geopotential amounts to ${ }^{7} 45 \%$. With this estimate the total error of the LAGEOS-LAGEOS II Lense-Thirring experiment would be of the order of $50 \%$. If the sum of the absolute values of the individual errors is considered, an upper bound of $83 \%$ on the systematic error due to the even zonal harmonics is obtained; the total error in the LAGEOS-LAGEOS II Lense-Thirring experiment would, then, amount to almost $100 \%$. It must be considered that, since in EGM96 a strong correlation among the various recovered even zonal coefficients does occur, the approach of the sum of the absolute values of the individual errors would, probably, yield a more realistic estimate of this kind of systematic error.

\subsection{The supplementary orbital planes option}

The originally proposed LARES mission ${ }^{9}$ consists of the launch of a LAGEOS-type satellite-the LARES-with the same orbit of LAGEOS except for the inclination $i$ of its orbit, which should be supplementary to that of LAGEOS, and the eccentricity $e$, which should be one order of magnitude larger. The choice of the particular value of the inclination for LARES is motivated by the fact that in this way, by using as observable the sum of the nodes of LAGEOS and LARES

$$
\Sigma \delta \dot{\Omega} \equiv \dot{\Omega}^{\mathrm{LAGEOS}}+\delta \dot{\Omega}^{\mathrm{LARES}}
$$

it should be possible to cancel out, to a high degree of accuracy, all the contributions of the even zonal harmonics of the geopotential, which depends on cos $i$, and add up the Lense-Thirring precessions which, instead, are independent of $i$. The systematic error due to the even zonal coefficients of geopotential would range from ${ }^{10} 0.8 \%$ to $4.6 \%$ for an uncertainty in $i_{\text {LARES }}$ up to $1 \mathrm{deg}$ with respect to its nominal values of Table 1, according to the variance matrix of EGM96 used in a root-sum-square fashion. If, instead, the sum of the absolute values of the individual errors is considered, the systematic error due to the even zonal coefficients of geopotential would amount to almost $2 \%$ for $i_{\text {LARES }}=70 \mathrm{deg}$ and to $10 \%$ for a departure of $i_{\text {LARES }}$

\footnotetext{
${ }^{\mathrm{d}}$ Such approach is considered more realistic by some authors ${ }^{8}$ because nothing would assure that the correlations among the even zonal harmonics of the covariance matrix of the EGM96 model, which has been obtained during a multidecadal time span, would be the same during an arbitrary past or future time span of a few years as that used in the LAGEOS-LAGEOS II Lense-Thirring experiment or in the proposed LAGEOS-LARES mission. Moreover, in the same paper it is claimed that a correct and reliable evaluation of the impact of the non-gravitational perturbations of the perigee of LAGEOS II on the proposed measurement is rather troublesome.
} 
of $1 \mathrm{deg}$ from its nominal value. The impact of the non-gravitational perturbations would be far less important because the nodes of the LAGEOS-like satellites are almost insensitive to them.

Since the Lense-Thirring precession of the pericentre depends on $\cos i$ and the classical precessions due to the even zonal harmonics of geopotential depend on $\cos ^{2} i$ and odd powers of $\sin i$, also the difference of the perigees ${ }^{11,12}$

$$
\Delta \dot{\omega} \equiv \delta \dot{\omega}^{\mathrm{S} 1}-\delta \dot{\omega}^{\mathrm{S} 2},
$$

of a pair of satellites with identical orbits in supplementary orbital planes could be used, in priciple, in order to measure the Lense-Thirring effect. It has been shown that, for a pair of LAGEOS-like satellites S1 and S2 (see Table 1) with the same physical properties of the LAGEOS satellites, the total error would not be less than ${ }^{12} 5 \%$, mainly due to the non-gravitational perturbations.

\subsection{Other approaches}

In order to cope with the problem of the impact of the unavoidable orbital injection errors in the inclination of LARES on the systematic error due to the even zonal harmonics of geopotential, the following observable has also been proposed ${ }^{13}$

$$
\delta \dot{\Omega}^{\mathrm{LAGEOS}}+c_{1} \delta \dot{\Omega}^{\mathrm{LAGEOS} \mathrm{II}}+c_{2} \delta \dot{\Omega}^{\mathrm{LARES}}+c_{3} \delta \dot{\omega}^{\mathrm{LARES}} \sim 61.8 \mu_{\mathrm{LT}},
$$

with

$$
c_{1} \sim 3 \times 10^{-3}, c_{2} \sim 9.9 \times 10^{-1}, c_{3} \sim 1 \times 10^{-3} .
$$

It would allow to neglect the problem of the correlations between the various even zonal coefficients of geopotential. Moreover, the secular variations in the even zonal harmonic coefficients of geopotential would not affect such observable. The same would also hold for the bias of the Lense-Thirring effect itself on the even zonal harmonics because it is mainly concentrated in $J_{2}$ and $J_{4}$. Notice that such an observable does not include the perigee of LAGEOS II.

Another possible way to overcome the uncertainties related to the error budget evaluation due to the non-gravitational perturbations which affects the perigee of LAGEOS II by using the existing satellites is the following combination ${ }^{14}$

$$
\delta \dot{\Omega}^{\mathrm{LAGEOS}}+c_{1} \delta \dot{\Omega}^{\mathrm{LAGEOS} \mathrm{II}} \sim 48.2 \mu_{\mathrm{LT}}, c_{1}=0.546
$$

A similar approach is implicitly presented in ref. ${ }^{15}$. The observable of eq. (9) would be affected neither by the secular variations in the $J_{\ell}$ coefficients nor, at least to a certain extent, by the a priori Lense-Thirring bias of the even zonal harmonics.

\subsection{The role of the various Earth gravity models}

Here we wish to discuss the impact of the new, forthcoming Earth gravity models from the CHAMP ${ }^{16}$ and GRACE $^{15}$ missions, which should greatly improve our knowledge of the terrestrial gravitational field, on the different combinations used 
or proposed for the measurement of the Lense-Thirring effect. Very recently, the EIGEN2 ${ }^{17}$, GRACE01S ${ }^{18}$ and GGM01C/Se models from the CHAMP and GRACE only data, respectively, have been released. Although very preliminary and lacking of reliable validation and calibration tests, they can be used in order to get an idea of which the improvements in the Lense-Thirring tests could be. In Table 2 we quote the sigmas of the normalized even zonal Stokes coefficients $\sigma_{\bar{C}_{\ell 0}}$ for EGM96, EIGEN2 and GRACE01S. Note that for the GRACE models the sigmas are not the formal errors but are approximately calibrated. For EIGEN2 the formal errors are released: it is likely that they are rather optimistic, at least for the low degree even zonal harmonics up to $\ell=20^{17}$. It should be noted that $J_{2}$ is known much less

Table 2. Errors in the even zonal normalized Stokes coefficients $\sigma_{\bar{C}_{\ell 0}}$ for various Earth gravity models up to degree $\ell=20$. The GGM01C model is very similar to GRACE01S, apart from $\sigma_{\bar{C}_{20}}=1.1677 \times 10^{-11}$ and $\sigma_{\bar{C}_{40}}=1.7874 \times 10^{-11}$.

\begin{tabular}{lccc}
\hline$\ell$ & EGM96 & EIGEN2 & GRACE01S \\
\hline 2 & $3.561 \times 10^{-11}$ & $1.939 \times 10^{-11}$ & $3.106 \times 10^{-10}$ \\
4 & $1.042 \times 10^{-10}$ & $2.230 \times 10^{-11}$ & $2.713 \times 10^{-11}$ \\
6 & $1.449 \times 10^{-10}$ & $3.136 \times 10^{-11}$ & $9.568 \times 10^{-12}$ \\
8 & $2.266 \times 10^{-10}$ & $4.266 \times 10^{-11}$ & $6.428 \times 10^{-12}$ \\
10 & $3.089 \times 10^{-10}$ & $5.679 \times 10^{-11}$ & $7.180 \times 10^{-12}$ \\
12 & $4.358 \times 10^{-10}$ & $7.421 \times 10^{-11}$ & $5.022 \times 10^{-12}$ \\
14 & $5.459 \times 10^{-10}$ & $9.561 \times 10^{-11}$ & $4.376 \times 10^{-12}$ \\
16 & $5.313 \times 10^{-10}$ & $1.216 \times 10^{-10}$ & $3.805 \times 10^{-12}$ \\
18 & $4.678 \times 10^{-10}$ & $1.532 \times 10^{-10}$ & $4.412 \times 10^{-12}$ \\
20 & $4.690 \times 10^{-10}$ & $1.914 \times 10^{-10}$ & $3.571 \times 10^{-12}$ \\
\hline
\end{tabular}

accurately in GRACE01S than in EGM96 and EIGEN2.

\section{Combinations with the existing or proposed geodetic satellites of LAGEOS-type}

The classical secular precessions of the node and the perigee, for a given even zonal harmonic of degree $\ell$, are proportional to $R^{\ell} a^{-\left(\frac{3+2 \ell}{2}\right)}$, where $R$ is the Earth mean equatorial radius. Then, the relevant classical secular precessions of the node and the perigee of the LAGEOS satellites, due to their high altitude (see Table 1), are those induced just by the first few five-six even zonal harmonics of geopotential in the sense that the error in the Lense-Thirring measurement due to geopotential does not change if the even zonal harmonics of degree higher than $\ell=10-12$ are neglected in the calculations.

eIt can be retrieved on the WEB at http://www.csr.utexas.edu/grace/gravity/ The GRACEonly GGM01S model was combined with the TEG-4 information equations (created from historical multi-satellite tracking data; surface gravity data and altimetric sea surface heights) to produce the preliminary gravity model GGM01C. 


\subsection{The full-range even zonal harmonics observables}

Let us recall that the sum of the nodes $\Sigma \dot{\Omega}$ of LAGEOS and LARES (and of S1 and S2), and the difference of the perigees $\Delta \dot{\omega}$ of S1 and S2 do not cancel any even zonal harmonic coefficients of geopotential due to the unavoidable orbital injection errors; in the case of the originally proposed LAGEOS-LARES mission this non perfect cancellation of the effects of the even zonal harmonics would occur even if the orbital parameters of LARES were exactly the same as in Table 1 because $e_{\text {LARES }}=0.04$, while $e_{\text {LAGEOS }}=0.0045$. This implies that it is of the utmost importance that the new gravity models yield notable improvements especially for $J_{2}, J_{4}$ and, to a lower extent, $J_{6}$ and $J_{8}$ which would affect such observables and which are the most relevant sources of systematic errors for the Lense-Thirring precessions of the nodes and the perigees of each satellite. This is also the case for the combination of eq. (9) which involves the node of LAGEOS and the node of LAGEOS II; it cancels out the first even zonal harmonic $J_{2}$ and is affected by the remaining $J_{4}, J_{6}, \ldots$

Let us consider the sum of the nodes of LAGEOS and LARES. By assuming an orbital injection error in $i_{\text {LARES }}$ up to $1 \mathrm{deg}$, it turns out that the variance matrix of EIGEN2 up to degree $\ell=20$ yields an error due to the even zonal harmonics of geopotential spanning from $0.2 \%$ to $1.6 \%$. GRACE01S, instead, induces an error which ranges from $0.2 \%$ to $24 \%$. The range error induced by GGM01C is $0.2 \%-0.9 \%$. Let us recall that the error due to EGM96 ranges from $2 \%$ to $^{\mathrm{f}} 10 \%$. These numbers can be explained if we consider that for large departures from the condition of ideal supplementary orbital planes the contributions of all the even zonal harmonics-and, especially, those of lower degrees-affect the systematic error due to geopotential. From Table 2 it can be noticed that $J_{2}$ is far better known in EIGEN2 than in GRACE01S. An analysis of the contributions of the various even zonal harmonics in the building up of the systematic error due to geopotential shows that for departures of $i_{\text {LARES }}$ of $1 \mathrm{deg}$ from its nominal value of Table 1 the most important role is played just by $J_{2}$. This also explains the better results obtainable with GGM01C.

The combination of eq. (9) is affected by the uncancelled even zonal harmonics $J_{4}$, $J_{6}, \ldots$ at a $177 \%, 22 \%, 21 \%$ and $14 \%$ level according to the sum of the absolute values of the errors of EGM96 and to the variance matrices of EIGEN2, GRACE01S and GGM01C, respectively. The small difference between the EIGEN2 and GRACE01S results can be explained by noticing that $J_{2}$ does not affect eq. (9), the error in $J_{4}$ is slightly better in EIGEN2 than in GRACE01S and the accuracy of the GRACE01S determinations of $J_{6}, J_{8}, \ldots$ is only slightly better than that of EIGEN2. The better results for GGM01C, especially for $J_{4}$, explain the result obtained with such Earth gravity model.

\footnotetext{
${ }^{\mathrm{f}}$ Note that for EIGEN2, GRACE01S and GGM01C a root-sum-square calculation with their variance matrices yields realistic estimates because, contrary to EGM96, their covariance matrices are almost diagonal.
} 


\subsection{The partial-range even zonal harmonics observables}

If, instead, we consider the suitably designed combinations of $N$ orbital residuals of the LAGEOS satellites which cancel out the first $N-1$ even zonal harmonics ${ }^{\mathrm{g}}$ the situation is different in the sense that the improvements of the remaining higher degree even zonal harmonics become relevant in order to reduce the systematic error due to the even zonal harmonics of geopotential which, for $N>3$ is well below $1 \%$ also with EGM96 (RSS calculation).

Let us consider the currently used observable of eq. (3) which cancels out $J_{2}$ and $J_{4}$. According to the variance matrices of EGM96, EIGEN2 and GRACE01S up to degree $\ell=20$ the systematic error due to geopotential amounts to $83 \%$ (sum of the absolute values of the errors), $9 \%$ and $2 \%$ (root-sum-square calculations), respectively. This shows the importance of reducing the uncertainties in $J_{6}$ and, to a lower extent, $J_{8}$.

The combination of eq.(7) is affected by the remaining even zonal harmonics $J_{8}$, $J_{10}, \ldots$ far less than $1 \%$. Indeed, for a maximum orbital injection error in $i_{\text {LARES }}$ of $1 \mathrm{deg}$ the systematic error due to geopotential amounts to $2.2 \%-3 \%$ according to the sum of the absolute values of the errors in the variance matrix of EGM96 up to degree $\ell=20$, to $0.08 \%-0.1 \%$ according to the variance matrix of EIGEN2, used in a root-sum-square fashion, up to degree $\ell=20$ and to $0.007 \%-0.01 \%$ according to the variance matrix of GRACE01S, used in a root-sum-square fashion, up to degree $\ell=20$. This can be explained by noticing from Table 2 that the higher degree even zonal harmonics are far better determined in GRACE01S (and GGM01C/S) than in the other Earth gravity models.

\section{Combinations with the other existing geodetic satellites}

In principle, a very appealing possibility would be the use of combinations which include also the orbital elements of the other existing geodetic satellites like Ajisai, Starlette, Stella, WESTPAC1, ETALON1, ETALON2, apart from those of LAGEOS and LAGEOS II; this option has been studied in some previous works ${ }^{19}$. In Table 3 the orbital elements of the other existing SLR satellites are reported. For

Table 3. Orbital parameters of Ajisai, Starlette, Stella, WESTPAC1, ETALON1 and ETALON2

\begin{tabular}{lcccccc}
\hline Orbital parameter & Ajisai & Starlette & Stella & WESTPAC1 & ETALON 1 & ETALON2 \\
\hline$a(\mathrm{~km})$ & 7870 & 7331 & 7193 & 7213 & 25498 & 25498 \\
$e$ & 0.001 & 0.0204 & 0 & 0 & 0.00061 & 0.00066 \\
$i(\mathrm{deg})$ & 50 & 49.8 & 98.6 & 98 & 64.9 & 65.5 \\
\hline
\end{tabular}

example, one might think about some combinations including only the nodes of the LAGEOS satellites and of some of the other geodetic SLR satellites. Unfortunately,

gWe assume $N>2$. 
it turns out that this way is unpracticable because the other satellites orbit at lower altitudes than LAGEOS and LAGEOS II (see Table 3), so that they are sensitive to much more even zonal harmonics, especially Starlette, Stella and WESTPAC1. Also EIGEN2 and GRACE01S do not alter this situation in a significative way. Indeed, it turns out that, while the systematic error due to the even zonal harmonics of geopotential of a combination which includes the nodes of LAGEOS and LAGEOS II, the perigee of LAGEOS II and the node of Ajisai is sensitive only to the harmonics up to degree $\ell=14$, according to the variance matrix of GRACE01S, for another combination similar to the previous one, with the node of Starlette instead of the perigee of LAGEOS II, the error induced by geopotential is sensitive well up to degrees $\ell=40-50$. Moreover, the calculation of the classical secular nodal precessions for degrees as high as $\ell=40$ is rather unreliable because of instabilities of the numerical results. However, it may be interesting to consider the following combination

$$
\delta \dot{\Omega}^{\mathrm{LAGEOS}}+c_{1} \delta \dot{\Omega}^{\mathrm{LAGEOS} \mathrm{II}}+c_{2} \delta \dot{\Omega}^{\mathrm{Ajisai}}+c_{3} \delta \dot{\Omega}^{\text {Starlette }}+c_{4} \delta \dot{\Omega}^{\text {Stella }} \sim 57.4 \mu_{\mathrm{LT}},
$$

with $c_{1}=4.174, c_{2}=-2.705, c_{3}=1.508, c_{4}=-0.048$. According to a RootSum-Square calculation with the variance matrix of GGM01C up to ${ }^{\mathrm{h}} \ell=42$ the impact of the remaining even zonal harmonics of degree $\ell \geq 10$ amounts to 21.6 mas $\mathrm{yr}^{-1}$ which yields a $37.6 \%$ percent error due to geopotential in the measurement of the Lense-Thirring effect with eq. (10). The upper bound due to the sum of the absolute values of the individual errors amounts to $123 \%$. If the future GRACEbased gravity solutions will improve the high degree $\left(J_{10}, J_{12}, J_{14}, \ldots\right)$ even zonal harmonics more than the low degree $\left(J_{2}, J_{4}, J_{6}, J_{8}\right)$ ones, the combination of eq.(10) might deserve some interest in alternative to that of eq. (9).

In conclusion, also with the new forthcoming Earth gravity models, it is unlikely that the combinations involving the orbital elements of the other existing geodetic satellites as well will represent a real alternative to those which use only the existing LAGEOS satellites and the proposed LARES. If, instead, we refer to the existing LAGEOS satellites only, it may happen that the inclusion of the nodes of the other geodetic satellites could become a viable possibility.

\section{Conclusions}

The choice of the optimal observable for measuring the Lense-Thirring effect is dictated by the need of getting a compromise among the reduction of the systematic gravitational error due to geopotential, the systematic non-gravitational error induced by the perturbations affecting especially the perigees of the geodetic SLR satellites and the reliability of the SLR data of the satellites orbits.

${ }^{\mathrm{h}}$ It has been checked that the error due to the even zonal harmonics remains stable if other even zonal harmonics are added to the calculation. Moreover, it turns also out that, up to $\ell=42$, there are no appreciable fluctuations in the calculated classical secular precessions. 
In regard to those requirements, it turns out that even with the new, forthcoming Earth gravity models the multisatellite approach involving the orbital elements of the existing SLR satellites other than the LAGEOS ones should not be competitive with the observables built up with the LAGEOS-type satellites only.

Among them, notice that the total accuracy obtainable with the originally proposed combination by Ciufolini, which involves the nodes of both LAGEOS and LAGEOS II and the perigee of LAGEOS II, strongly depends on the correct evaluation of the impact of the non-gravitational perturbations on the perigee of LAGEOS II because it is arguable that the error due to geopotential could be reduced to $1 \%$ or, perhaps, less in the near future. On the contrary, for the combination proposed explicitly in ref. ${ }^{14}$, which involves the nodes of LAGEOS and LAGEOS II, the improvements in the Earth gravity field even zonal coefficients, especially $J_{4}, J_{6}, J_{8}$, will be of crucial importance in reducing the total error to less than $10 \%$ because the impact of the non-gravitational perturbations on the error budget is quite negligible. Also a combination involving the nodes of LAGEOS, LAGEOS II, Ajisai, Starlette and Stella might become interesting, provided that notable improvements in the higher degree even zonal harmonics $J_{10}, J_{12}, \ldots$ will occur.

For the observables involving the proposed LARES, the multisatellite combination including also the elements of LAGEOS and LAGEOS II would not require any particular improvements in the knowledge of the higher degree even zonal harmonics of geopotential because the systematic error induced by them, according to the present-day level of knowledge of the terrestrial gravitational field, would be sufficiently small.

\section{References}

1. J. Lense and H. Thirring Phys. Z. 19, 156 (1918).

2. I. Ciufolini and J. A. Wheeler. Gravitation and Inertia (Princeton University Press, 1995).

3. L. Iorio Il Nuovo Cimento B 116, 777 (2001).

4. I. Ciufolini Class. Quantum Grav. 17, 2369 (2000).

5. I. Ciufolini Il Nuovo Cimento A 109, 1709 (1996).

6. F.G. Lemoine et al. The Development of the Joint NASA GSFC and the National Imagery Mapping Agency (NIMA) Geopotential Model EGM96, NASA/TP-1998-206861 (1998).

7. L. Iorio Celest. Mech and Dyn. Astron. 86, 277 (2003).

8. J. Ries, R.J. Eanes and B.D. Tapley, in Nonlinear Gravitodynamics. The LenseThirring Effect, ed. R. Ruffini, C. Sigismondi (World Scientific, Singapore 2003), p. 201.

9. I. Ciufolini, Phys. Rev. Lett. 56, 278, (1986); L. Iorio, D.M. Lucchesi and I. Ciufolini, Class. Quantum Grav. 19, 4311, (2002).

10. L. Iorio Gen. Rel. Grav. 35, 1263 (2003).

11. L. Iorio Phys. Lett. A 308, 81, (2003).

12. L. Iorio and D.M. Lucchesi Class. Quantum Grav. 20, 2477, (2003).

13. L. Iorio et al., "On the possibility of measuring relativistic gravitational effects with a LAGEOS-LAGEOS II-OPTIS-mission" http://www.arxiv.org/abs/gr-qc/0211013 
preprint, (2003).

14. L. Iorio and A. Morea, "The impact of the new Earth gravity models on the measurement of the Lense-Thirring effect" http://www.arxiv.org/abs/gr-qc/0304011 preprint, Gen. Rel. Grav., in press (2004).

15. J.C. Ries, R.J. Eanes, B.D. Tapley and G.E. Peterson, "Prospects for an Improved Lense-Thirring Test with SLR and the GRACE Gravity Mission" http://cddisa.gsfc.nasa.gov/lw13/lw_proceedings.html\#science preprint, (2002).

16. E. Pavlis, in Recent Developments in General Relativity ed. R. Cianci, R. Collina, M. Francaviglia and P. Fré (Milan, Springer 2000), p. 217.

17. Ch. Reigber et al., Adv. Sp. Res. 31, 1883, (2003).

18. Ch. Reigber et al., "First EIGEN Gravity Field Model based on GRACE Mission Data Only" Geophys. Res. Lett. to be submitted, (2003).

19. L. Iorio Class. Quantum Grav. 19, 5473, (2002). 